\title{
Effect of compatibilization on the breakup of polymeric drops in shear flow
}

\author{
Sachin Velankar, Peter Van Puyvelde, Jan Mewis, and Paula Moldenaers ${ }^{\text {a) }}$ \\ Department of Chemical Engineering, K.U. Leuven, de Croylaan 46, \\ B-3001 Leuven, Belgium
}

(Received 27 December 2000; final revision received 16 April 2001)

\begin{abstract}
Synopsis
A block copolymer may be added as a compatibilizer during polymer processing in order to promote intimate mixing of thermodynamically immiscible homopolymers. The action of this compatibilizer can only partially be attributed to its effect on the interfacial tension between the immiscible homopolymers. Here the additional contributions of the compatibilizer are directly probed by measuring the capillary number during coalescence experiments. Model blends consisting of polyisobutylene (PIB) and polydimethylsiloxane (PDMS), compatibilized with various amounts of a PIB-PDMS diblock copolymer, are used for this purpose. The mean capillary number of the droplets is determined from the mechanical frequency response of the blends. With increasing amounts of compatibilizer, a systematic increase in steady shear capillary number is seen, to values well above the critical capillary number for droplet breakup of uncompatibilized systems. This indicates that a simple decrease in interfacial tension is not the only effect of adding the compatibilizer to these immiscible blends. Past simulations suggest that these results are associated with gradients in interfacial tension (Marangoni stresses) induced by the gradients of compatibilizer concentration due to flow. Direct evidence of the presence of such interfacial tension gradients along the surface of compatibilized drops was obtained by optical microscopy. (C) 2001 The Society of Rheology. [DOI: 10.1122/1.1380424]
\end{abstract}

\section{INTRODUCTION}

Mechanical blending of immiscible polymers is a common means of achieving the desired material properties in polymer processing. The immiscible components are usually blended in the melt state and then cooled, thereby freezing the nonequilibrium, multiphase microstructure. The properties of the blend are strongly influenced by the size of heterogeneity, making the relationships between processing flow conditions and the resulting microstructure a topic of intense research. Restricting ourselves to two-phase droplet-matrix morphologies, the droplet size $R$ is governed primarily by a comparison between breakup and coalescence of droplets.

For the case of uncompatibilized droplets, breakup occurs if the hydrodynamic stress deforming the droplet is significantly larger than the restoring stress $\alpha / R$ due to interfacial tension $\alpha$. For a Newtonian droplet suspended in a Newtonian matrix of viscosity $\eta$, and being sheared at shear rate of $\dot{\gamma}$, the hydrodynamic stress is characterized by $\dot{\gamma} \eta$ and, hence, breakup occurs when [Grace (1982); Rallison (1984)]

\footnotetext{
a) Author to whom correspondence should be addressed. Electronic mail: paula.moldenaers@cit.kuleuven.ac.be
} 


$$
\mathrm{Ca}=\frac{\text { hydrodynamic stress }}{\text { interfacial stress }}=\frac{R \dot{\gamma} \eta}{\alpha} \geqslant \mathrm{Ca}_{\mathrm{cr}}(p)
$$

where $\mathrm{Ca}_{\mathrm{cr}}$ is the critical capillary number. $\mathrm{Ca}_{\mathrm{cr}}$ depends on $p$, the ratio of the viscosity of the droplet to that of the matrix, and has been shown to diverge above a viscosity ratio of approximately 4 [Grace (1982)]. For viscosity ratios close to 1 , as in this paper, $\mathrm{Ca}_{\mathrm{cr}}$ is known to be about 0.5 .

Coalescence is governed by the product of the collision frequency between droplets in shear flow, and an efficiency factor accounting for the fact that only a small fraction of collisions result in coalescence [Chesters (1991); Vinckier et al. (1998)]. The collision frequency, $C$, is given by the Smoluchowski equation

$$
C=\frac{16}{3} \dot{\gamma} R^{3} n^{2}
$$

where $n$ is the number density of droplets. At constant volume fraction of the droplet phase, $\phi$, the product $n R^{3}$ is constant, and hence, $C \propto n$, or $C \propto R^{-3}$.

Surface-active species are often employed to achieve a finer dispersion of droplets; this "compatibilizer" may be added prior to mixing (physical compatibilization) or be generated at the interface by a chemical reaction (reactive compatibilization) during mixing [di Lorenzio and Frigione (1997)]. In many cases, the compatibilizer is believed to reduce the interfacial tension, and hence, the interfacial restoring stress, and therefore reduce the droplet size as per Eq. (1). The view that effects of compatibilization are entirely attributable to a decrease in interfacial tension has been challenged by several observations such as coalescence suppression by steric hindrance [Sundararaj and Macosko (1995); Macosko et al. (1996), Milner and Xi (1996)], and interfacial viscoelasticity [Riemann et al. (1997)] due to added compatibilizer. However, the available data are too meager to allow a definitive conclusion about the effects of compatibilization in immiscible polymer blends. Moreover, most compatibilized blends studied so far were composed of high molecular weight commercial polymers. The high elasticity and shearthinning nature of the blend components complicate quantitative analysis of the result. This research seeks to clarify the effects of added compatibilizer on the dynamics of immiscible blends by using simple model blends as described below.

Several groups \{see [Guido et al. (1999)], and references therein\} have successfully avoided problems with complex rheological properties of the components of blends by studying morphological evolution in idealized polymer blends subjected to well-defined simple flows. These blends, usually based on polydimethylsiloxane (PDMS) and polyisobutylene (PIB) homopolymers, offer several advantages including simple rheological behavior of the components, low viscosities at room temperature, and optical transparency. In the present work, the effect of compatibilization has been studied on such a PIB/PDMS system by adding a diblock copolymer of PIB and PDMS to the blend. The diblock copolymer is expected to preferentially adsorb at the interface between the components and thereby act as a compatibilizer. Optical microscopy experiments have confirmed that the diblock copolymer is an effective compatibilizer for a PIB/PDMS blend, i.e., the average size of the droplets after a given shear history is significantly reduced by addition of the diblock copolymer to the blend. This paper, however, is only concerned with capillary numbers of droplets, and not with their size as such. The main advantage in dealing with $\mathrm{Ca}$ rather than droplet size is that the decrease in interfacial tension due to presence of compatibilizer is already accounted for in the definition of Ca. Thus, the question underlying the present approach is: What are the differences between the dy- 
namics of uncompatibilized and compatibilized droplet-matrix blends when the droplets of compatibilized blends are "normalized" for their lower interfacial tension?

\section{EXPERIMENT}

The blends studied here were composed of PDMS $\left(M_{w} 145000\right.$, Rhodorsil 47 vs 100000 from Rhodia Chemicals, $\eta=107 \mathrm{Pas}$ at $23^{\circ} \mathrm{C}$ ) as the matrix phase and PIB $\left(M_{w}\right.$ 1300, Parapol 1300 from Exxon Chemical, $\eta=113 \mathrm{Pas}$ at $23^{\circ} \mathrm{C}$ ) as the droplet phase. Both components were nearly Newtonian under experimental conditions. A diblock copolymer of PIB-PDMS $\left(M_{w, \mathrm{PIB}} \approx 6150 ; M_{w}\right.$,PDMS $\approx 8000$; polydispersity $\approx 1.3$ ) was purchased from Polymer Source Inc. The low molecular weights of the blocks imply that there are no entanglements between the blocks and the bulk phases.

Blends were prepared by mixing the diblock copolymer into PIB to obtain the dispersed phase, and then blending this mixture into the PDMS matrix. All mixing was performed by hand with a spatula. All samples were preconditioned with a specific shear history prior to measurement, and differences in initial mixing conditions were found to be relatively unimportant as also noted previously for similar uncompatibilized blends [Vinckier et al. (1998)]. All blends had 10\% by weight of the dispersed phase (volume fraction $=0.108$ ) unless noted otherwise. The amount of diblock is quoted as a weight percentage of dispersed phase. For example, a " $4 \%$ compatibilized" blend has overall $0.4 \%$ by weight of diblock copolymer, based on the global blend composition. Blends with $0 \%-10 \%$ compatibilizer were studied. The addition of a block copolymer in these relatively small amounts was found to have a negligible effect on the viscoelastic properties of the bulk phases.

Rheological measurements were performed on a Rheometrics Dynamic Stress Rheometer at $23.0{ }^{\circ} \mathrm{C}$ using a $25 \mathrm{~mm}$ diameter, $0.1 \mathrm{rad}$ cone and plate geometry. All samples were subjected to a preshear of $4.8 \mathrm{~s}^{-1}$ for 3000 strain units, and then allowed to coalesce at a constant shear rate of $1.2 \mathrm{~s}^{-1}$. The evolution of morphology was monitored by interrupting the shear flow periodically, and conducting dynamic mechanical measurements at $10 \%$ strain in the frequency range from 0.03 to $100 \mathrm{rad} / \mathrm{s}$. Each frequency sweep experimental lasted 1000-4000 s depending on the frequency at which the shoulder in $G^{\prime}$ appeared (see later). It was verified that measurements were in the linear viscoelastic region, and that increasing the preshear time at $4.8 \mathrm{~s}^{-1}$ did not affect the coalescence results significantly. In addition, repeated frequency sweep experiments over 7-8 $\mathrm{h}$ at the end of the preshear were found to give identical results, indicating that the morphology remained unchanged during frequency sweep experiments, and under quiescent conditions. This experimental strategy is unlikely to be successful at high volume fractions of the dispersed phase when static coalescence may cause substantial changes to the morphology over the time scales of the frequency sweep experiment [Macosko et al. (1996)].

\section{ANALYSIS OF DATA}

Figure 1 shows typical examples of the storage modulus $G^{\prime}$ of blends with various levels of compatibilizer. These data are chosen at various shearing times after stepping down the shear rate to $1.2 \mathrm{~s}^{-1}$ such that the shoulder appears at roughly comparable frequencies for all three samples. The high frequency part of the storage modulus $G^{\prime}$ can be described well by the Dickie model [Dickie (1973)], or equivalently, by the Palierne model [Palierne (1990)] without interfacial tension $(\alpha=0)$. The high-frequency values of $G^{\prime}$ are also approximately equal to the volume average modulus of the components (not shown). At lower frequencies, the blends show a pronounced shoulder in $G^{\prime}$ which, in the case of uncompatibilized blends, can be related to the shape-relaxation of deformed 


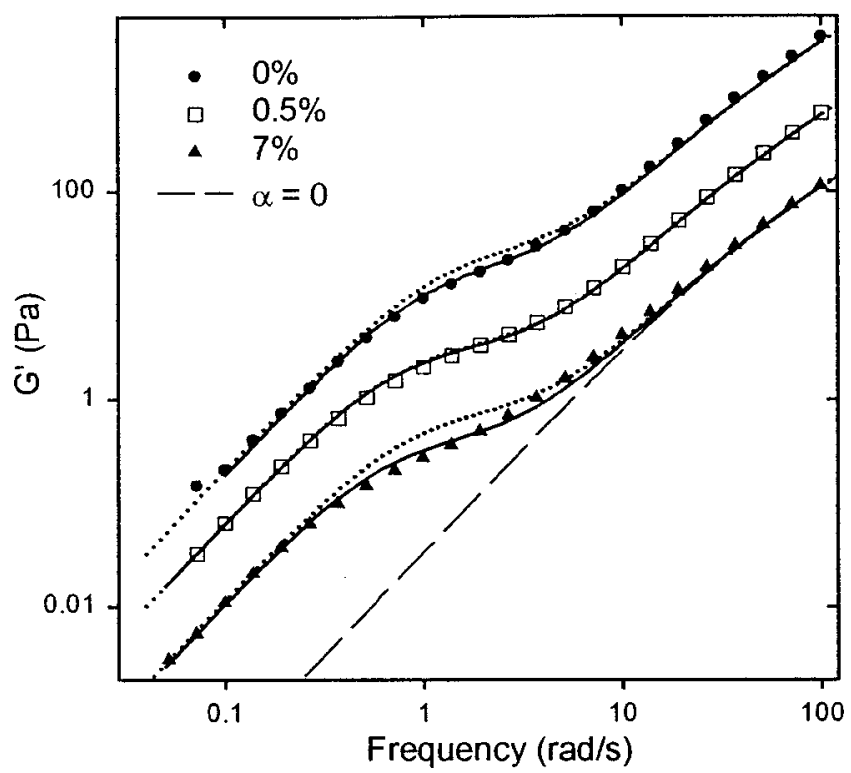

FIG. 1. Typical dynamic data for blends with $10 \%$ by weight of dispersed phase, and various amounts of compatibilizer. Dotted lines are fits to Palierne model with $\phi=0.108$. Solid lines allow $\phi$ to vary freely. The $y$-scale refers to the $0 \%$ data; all successive curves are shifted downwards by a factor of 5 for clarity. The lowermost dashed curve shows the $G^{\prime}$ expected for the $7 \%$ compatibilized blend if the interfacial tension were zero.

droplets [Palierne (1990); Graebling et al. (1993)]. This storage of mechanical energy due to deformation of the interface is a crucial feature of the dynamics of immiscible blends. Palierne [Palierne (1990); Graebling et al. (1993)] has related the mechanical frequency response of the blend to the characteristic interfacial stress $\alpha / R$ of the droplets by an exact analysis of a dilute dispersion of droplets under small amplitude deformation. Several studies [Gramespacher and Meissner (1992); Graebling et al. (1994); Lacroix et al. (1997); Vinckier et al. (1998)] on uncompatibilized blends have verified the validity of the Palierne model by demonstrating that the value of the parameter $\alpha / R$ required to fit the measured moduli of droplet-matrix morphologies is consistent with independent estimates of $\alpha$ and $R$.

The dotted lines in Fig. 1 show the results of fitting the Palierne model to the frequency sweep data, with $\alpha / R$ as the only fitting parameter. While qualitative features of the data can be fitted well, the model overpredicts the intensity of the shoulder for the uncompatibilized blends. Such overpredictions have been observed previously in similar uncompatibilized blends [Vinckier et al. (1996)]. Interestingly enough, with the addition of a diblock copolymer to the blend, the predictions improve for $0.5 \%$ and $1 \%$ compatibilization (1\% not shown). The fits then worsen appreciably with increasing amounts of compatibilizer (e.g., the 7\% compatibilized sample in Fig. 1). There are at least three possible reasons for these relatively poor fits: the Palierne model is not valid when a block copolymer is present on the interface, hydrodynamic interactions between droplets are not negligible at $10.8 \%$ by volume of the dispersed phase, and finally, polydispersity in $\alpha / R$ is large. Obviously, the first reason cannot explain the discrepancy for the uncompatibilized case, nor can it explain the improved fit at low levels of compatibilizer. Second, hydrodynamic interactions were not included in the fitting procedure because thus far, the Palierne model has not been extended to include hydrodynamic interactions. 


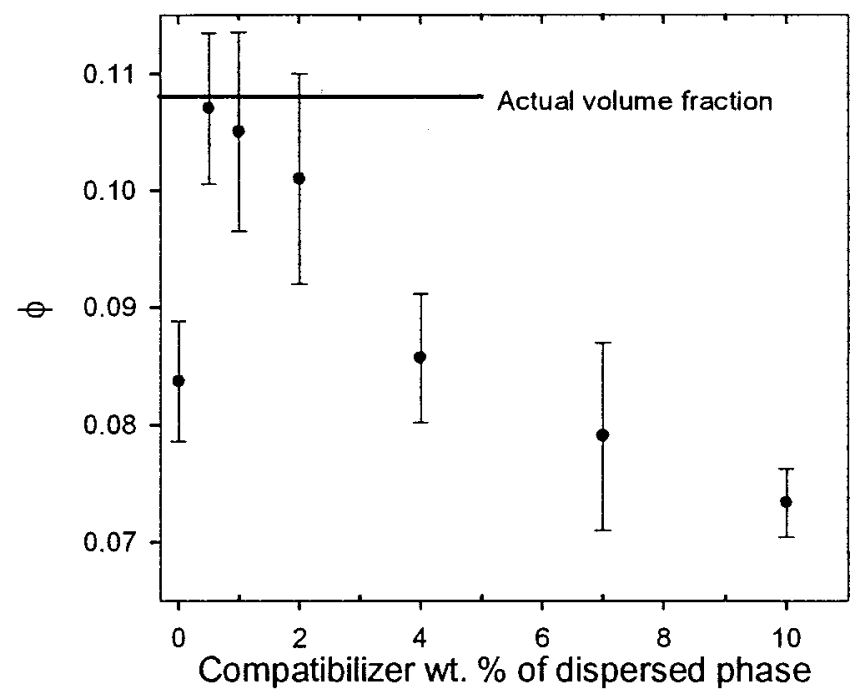

FIG. 2. Volume fractions of dispersed phase required for variable-volume-fraction fits to Palierne model.

In any case, all blends studied here have the same volume fraction of dispersed phase, and hydrodynamic interactions may be expected to be comparable. Finally, small amounts of polydispersity have been shown to have very little effect on the frequency response [Graebling et al. (1993); Vinckier et al. (1998)]. However, we have no means of demonstrating that polydispersity in $\alpha / R$ for the compatibilized blends is small. Note that in the present situation, unlike in uncompatibilized blends, polydispersity in drop size is not the same as polydispersity in $\alpha / R$ since the concentration of the compatibilizer, and hence, the interfacial tension, may vary from drop to drop. Given the situation, how may the frequency response be related to a mean $\alpha / R$ ?

Three methods were considered here. The first involved fitting the Palierne model to the data with $\alpha / R$ as th only free parameter. This method has been used previously to fit the $G^{\prime}$ of uncompatibilized blends, and thus obtain the mean droplet size using the known interfacial tension [Lacroix et al. (1997); Vinckier et al. (1998)]. As mentioned earlier, typical fits resulting from this procedure are shown as dotted lines in Fig. 1. This method resulted in accurately fitting the low and high frequencies, but generally overpredicting the $G^{\prime}$ at the shoulder, and somewhat underestimating the relaxation time of the shoulder. The corresponding capillary number $\eta \dot{\gamma} R / \alpha$ will be denoted by Ca.

Recent experiments [Lyu et al. (2000)] show that in an uncompatibilized dropletmatrix blend under shear, the smallest droplets tend to follow the streamlines around the larger ones and hence coalesce at much lower rates than the larger ones. Thus, after long periods of shearing, a certain fraction of droplets are far smaller than average, and thus have relaxation times far from the average. This suggests using a fitting method that explicitly accounts for the fact that a fraction of the droplets do not contribute to the shoulder in $G^{\prime}$. Hence, the second method used for fitting $G^{\prime}$ allowed $\alpha / R$ and the volume fraction $\phi$ of the dispersed phase to vary simultaneously. This procedure resulted in excellent fits throughout the frequency range for all samples as seen by the solid lines in Fig. 1. Figure 2 shows that the volume fractions of dispersed phase required for these fits range from $\phi \approx$ (actual volume fraction of 0.108 ) for 0.5 and $1 \%$ compatibilization, to $\phi \approx 0.075$ at high compatibilizer concentrations. For all samples these best-fit volume fractions remained almost unchanged as coalescence proceeded, implying that the vol- 


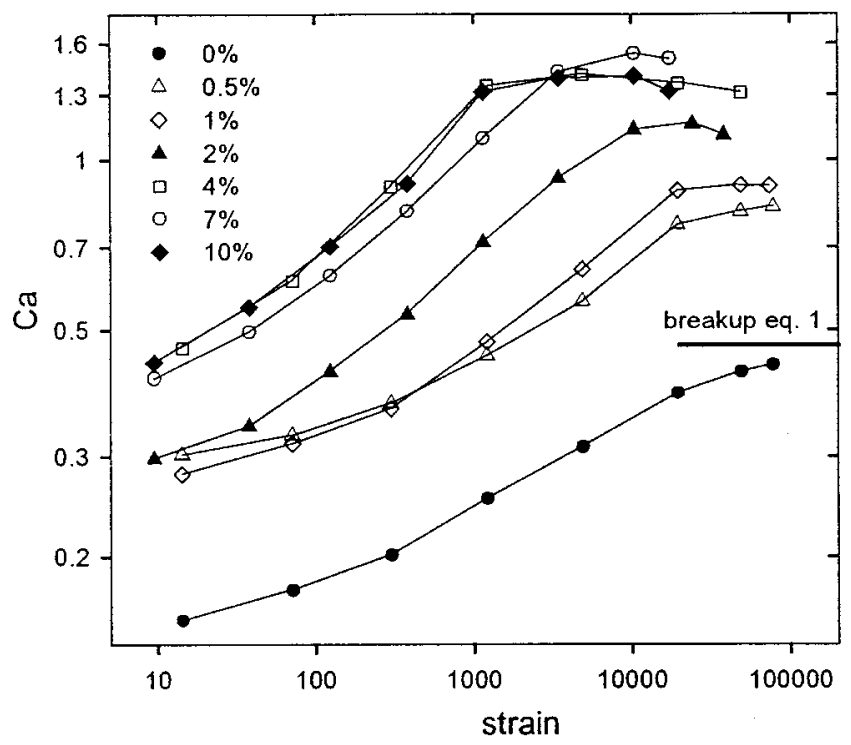

FIG. 3. Evolution of capillary number Ca during coalescence at $1.2 \mathrm{~s}^{-1}$ after preshearing at $4.8 \mathrm{~s}^{-1}$. The legend shows the amount of compatibilizer as a weight fraction of the dispersed phase.

ume fraction of droplets contributing to the shoulder remained unchanged during the coalescence process. The capillary numbers so obtained, denoted by $\mathrm{Ca}_{\phi}$, were up to $40 \%$ higher than $\mathrm{Ca}$, with the largest differences occurring at the early stages of coalescence at short shearing times. After long shearing times, the differences between $\mathrm{Ca}$ and $\mathrm{Ca}_{\phi}$ were much smaller (see later, and Fig. 4 for example).

Finally in the last fitting method used, the Palierne model was ignored altogether. The only assumption made was that the shoulder in $G^{\prime}$ represents the shape relaxation of the droplets. The shoulder relaxation time $\tau_{d}$ was then determined by fitting a Maxwell model to the difference between the measured $G^{\prime}$ of the blend, and the $G^{\prime}$ expected with $\alpha=0$ [Dickie (1973)]. A model-independent capillary number was then defined as $\tau_{d} \dot{\gamma}$. This was found to be a factor of 2.5-3 higher than $\mathrm{Ca}_{\phi}$ for all samples, which is in reasonable agreement with a factor of about 2.4 calculated from the Palierne model prediction

$$
\frac{\text { Droplet relaxation time }}{R \eta / \alpha}=\frac{4[10(p+1)-2 \phi(5 p+2)]}{(19 p+16)[2 p+3-2 \phi(p-1)]}
$$

using $p=1.06$ and values of $\phi$ from Fig. 2.

The model independent capillary number $\left(\tau_{d} \dot{\gamma}\right)$, and $\mathrm{Ca}_{\phi}$ show identical trends since they both fit the shoulder in $G^{\prime}$ very well, and hence, the former will not be shown here. Steady state values of both $\mathrm{Ca}$ and $\mathrm{Ca}_{\phi}$ will be presented later, however, the evolution of capillary number during coalescence will be shown only for $\mathrm{Ca}$. The conclusions drawn in this paper are independent of the fitting method used; it is dependent only on the assumption that the shoulder in $G^{\prime}$ corresponds to the shape-relaxation of droplets.

\section{RESULTS}

Figure 3 shows the evolution of Ca during coalescence at a shear rate of $1.2 \mathrm{~s}^{-1}$ for samples with various levels of compatibilizer. Coalescence is seen to increase the capil- 


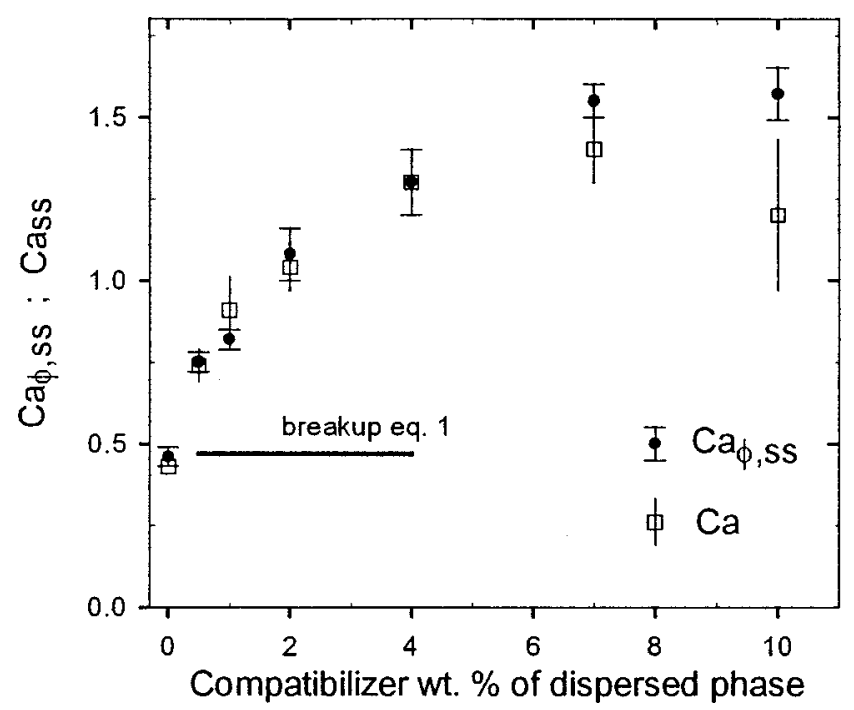

FIG. 4. Steady state capillary numbers $\mathrm{Ca}$ and $\mathrm{Ca}_{\phi}$ at $1.2 \mathrm{~s}^{-1}$. Each point is an average of three experiments on independently mixed samples.

lary number from an initial low value to a higher steady state value at all the compatibilizer concentrations studied. For the uncompatibilized sample, the final steady state capillary number $\mathrm{Ca}_{\mathrm{ss}}$ is seen to approach approximately 0.45 after long shearing times. This value is just below the critical capillary number for breakup, $\mathrm{Ca}_{\mathrm{cr}} \approx 0.5$, expected at the viscosity ratio of 1.06 [Grace (1982); Rallison (1984)]. Thus, following Vinckier et al.(1998), it may be concluded that the droplets grow by coalescence until they reach the critical capillary number, after which there exists a dynamic equilibrium between breakup and coalescence.

Upon adding compatibilizer, $\mathrm{Ca}$ is found to be considerably higher than for the uncompatibilized blend at all shear strains. Specifically, $\mathrm{Ca}_{\mathrm{ss}}$ for the compatibilized blends is found to be well above 0.5 , which is the $\mathrm{Ca}_{\mathrm{cr}}$ for breakup of uncompatibilized droplets. Thus, the addition of a surface-active compatibilizer allows the droplets to sustain a higher capillary number without breakup, which is the central result of this paper. This implies that in a dimensionless sense, the compatibilized droplets studied in the paper are more stable against breakup than uncompatibilized ones.

The major advantage of measuring relaxation of droplets is that capillary numbers can be obtained directly, rather than by combining separate measurements of drop size and interfacial tension. As mentioned in Sec. I, presenting data in the form of capillary number corresponds to normalizing the size of the droplets of a compatibilized blend for their lower interfacial tension. The increase in the capillary number with the addition of a compatibilizer clearly shows that the effect of compatibilization cannot be captured in terms of a decrease in interfacial tension alone, i.e., the scaling implied by Eq. (1) is not valid for the blends studied in this paper. Specifically, the higher capillary number of these compatibilized droplets indicates that the decrease in steady-shear drop size due to addition of compatibilizer is less than expected from the decrease in interfacial tension.

Figure 4 summarizes the steady shear capillary numbers $\mathrm{Ca}_{\mathrm{ss}}$ (from Fig. 3), and $\mathrm{Ca}_{\phi, \mathrm{s}}$, at $1.2 \mathrm{~s}^{-1}$ as a function of compatibilizer content. It is seen that $\mathrm{Ca}$ and $\mathrm{Ca}_{\phi, \mathrm{ss}}$ are almost equal up to about $4 \%$ compatibilization. $\mathrm{Ca}$ is seen to decrease at higher compatibilization. This is, however, an artifact of the poor quality of the Palierne fits for blends 
with large amounts of compatibilizer, which result in underestimating the relaxation time of the shoulder in $G^{\prime}$. On the other hand, $\mathrm{Ca}_{\phi, \mathrm{ss}}$ and $\tau_{d} \dot{\gamma}$ (not shown), which estimate the relaxation time of the shoulder more accurately, show a plateau above about $4 \%$ compatibilization. The plateau in $\mathrm{Ca}_{\phi, s s}$ indicates that the effects of a compatibilizer, other than a simple decrease in interfacial tension, saturate at high compatibilizer concentrations.

A final caution with regards to interpretation of Fig. 3 is that for the uncompatibilized blend, the increase in $\mathrm{Ca}$ with strain translates directly into a proportionate increase in droplet size as per Eq. (1). This may not be true for the compatibilized blends since the interfacial tension may itself change as coalescence proceeds. Specifically, if almost all the block copolymer remains on the surface of droplets at all times, the decrease in interfacial area must cause a decrease in interfacial tension as drops grow by coalescence. In that case, the relative increase in capillary number by coalescence is expected to exceed the relative increase in droplet size.

\section{DISCUSSION}

The main result of this paper is that in the blends studied here, the addition of surfaceactive compatibilizer leads to an increase in the steady shear capillary number to values well above the $\mathrm{Ca}_{\mathrm{cr}}$ required for breakup of uncompatibilized droplets. This discussion seeks to provide a microscopic basis to explain this observation.

Two prior publications have described such an increase in $\mathrm{Ca}_{\mathrm{cr}}$ due presence of a surface-active species, although both these involved microscopic observation of singledrop deformation and not droplet-matrix blends. The most explicit observation has been by Hu et al. (2000), who generated a block copolymer by interfacial reaction and obtained the interfacial tension by measuring drop deformation in extensional flow. These authors noted that, under certain conditions, the critical capillary number required for breakup of drops with block copolymer exceeded that for drops without block copolymer. A similar result is also implicit in the data of Janssen et al. (1994) for drops in shear flow, although in this case the surfactant was soluble in the bulk solution and the results were complicated by diffusive exchange of the surfactant between the interface and the bulk.

The situation has, however, been studied extensively by numerical simulation of drops with an interfacial tension-reducing surfactant on the interface [Stone and Leal (1990); Milliken and Leal (1994); Li and Pozrikidis (1997)]. The various phenomena involved are understood most easily for a drop in purely extensional flow [Stone and Leal (1990)]. In this case, the bulk flow in and around the extended drop convects the surfactant towards the end of the drop. The increased surfactant concentration at the ends of the drop reduces the local interfacial tension resulting in two competing effects: The lower interfacial tension at the ends of the drop encourages larger deformation, but the gradient in interfacial tension induces a Marangoni stress along the drop surface which opposes deformation. If diffusivity of the surfactant along the drop surface is large, concentration gradients, and hence, interfacial tension gradients, are negligible. In this case, the increase in the surface area of the drop due to deformation reduces the concentration of surfactant everywhere on the surface. This "overall dilution" of surfactant raises the average interfacial tension of the drop, and hence, opposes deformation. The net effect on the deformation and on $\mathrm{Ca}_{\mathrm{cr}}$ is an interplay of these effects. Additional complexity is introduced by diffusion of surfactant between the surface and the bulk [Milliken and Leal (1994)], and by droplet rotation in the case of shear flows [Li and Pozrikidis (1997)].

The importance of the diffusion of the block copolymer along the interface, and diffusion into the bulk may be estimated as follows. In the present case, since the diblock 
copolymer is unentangled, its diffusivity along the drop surface, $D_{s}$, is at most equal to the diffusivity of pure PIB with $M_{w}=6150$ (the block $M_{w}$ ) [Dalvi et al. (1993)]. Because the monomeric friction of PDMS is four orders of magnitude lower than that of PIB [Ferry (1980)], the PDMS block is expected to have a negligible effect on the diffusivity along the drop surface. Thus, the upper limit for $D_{s}$ is given by the Rouse theory

$$
D_{s}=\frac{R T}{N \zeta N_{\mathrm{Avo}}}
$$

where $\zeta$ is the monomeric friction coefficient of PIB, $N$ is the number of monomers in each PIB block, and $N_{\text {Avo }}$ is Avogadro's number. Using $\zeta=4.5 \times 10^{-8} \mathrm{~kg} / \mathrm{s}$ [Ferry (1980)], the upper limit for $D_{s}$ is found to be $\sim 10^{-15} \mathrm{~m}^{2} / \mathrm{s}$. Experiments with optical microscopy have shown that drop diameters can range from $10 \mu \mathrm{m}$ to less than the resolution of an optical microscope $(\sim 1 \mu \mathrm{m})$. Thus, with $D_{s}=10^{-15} \mathrm{~m}^{2} / \mathrm{s}$, the Peclet numbers $\left(\mathrm{Pe}=\dot{\gamma} R^{2} / D_{s}\right)$ span a range from 30000 to 3, arbitrarily assuming that smallest drops have a diameter of $0.1 \mu \mathrm{m}$. Since surface diffusion is much faster than convection only when Pe $\ll 1$ [Stone and Leal (1990); Li and Pozrikidis (1997)], the effects of overall dilution of surfactant mentioned in the previous paragraph may be ruled out. Diffusivity of the diblock perpendicular to the interface is expected to be significantly lower than $D_{s}$ since bulk diffusion involves pulling the PIB block through the PDMS matrix or vice versa [Dalvi et al. (1993)]. Therefore, the diffusive exchange of the block copolymer with the bulk is also expected to be negligible and the amount of diblock per droplet may be regarded as invariant in deformations not involving coalescence or breakup. Thus, having eliminated overall dilution and bulk diffusion as significant factors, Marangoni stresses remain the most likely explanation for the increase in $\mathrm{Ca}_{\mathrm{Cr}}$ with addition of block copolymer within the framework of theory [Stone and Leal (1990); Li and Pozrikidis (1997)].

Optical microscopy lends support to the physical picture described earlier. These experiments were conducted on blends composed of the same components as earlier, but with only $1 \%$ by weight of the dispersed phase in a PDMS matrix. This lower volume fraction of dispersed phase was necessary because the strong light scattering by the droplets makes the blends with $10 \%$ dispersed phase highly opaque. The amounts of compatibilizer studied were $0 \%, 2 \%$, and $10 \%$ by weight of the dispersed phase as before. The blends with $1 \%$ dispersed phase were subjected to the following shear history: they were first sheared at $0.1 \mathrm{~s}^{-1}$ for a sufficiently long time such that the droplets coalesced to a size that was easily visible under microscope. Shear was then stopped, allowing the droplets to retract to spherical shape. The blends were then sheared for $2 \mathrm{~s}$ at $10 \mathrm{~s}^{-1}$ to deform the droplets. The shearing was then stopped, and the subsequent relaxation of the droplets was observed. For the uncompatibilized blend, shear at $10 \mathrm{~s}^{-1}$ deformed the droplets into highly extended fibrils. After cessation of shear, these fibrils broke up by Rayleigh instabilities into strings of several smaller droplets as observed previously in a similar blend [Mewis et al. (1998)]. Droplets of the 2\% compatibilized blend were found to deform much less during the $10 \mathrm{~s}^{-1}$ shear, and highly extended fibrils were not seen. Figure 5 shows the relaxation of this blend $2 \mathrm{~s}$ after cessation of shear. It is seen that several droplets have severely extended tips, which were observed to retract much more slowly than the midsection of the droplets. The high curvature of the interface at the tips of the drops as compared to the midsection and the slow rate of tip retraction prove that the tips have a far lower interfacial tension than the midsection. This figure provides direct evidence of the presence of gradients in interfacial tension along 


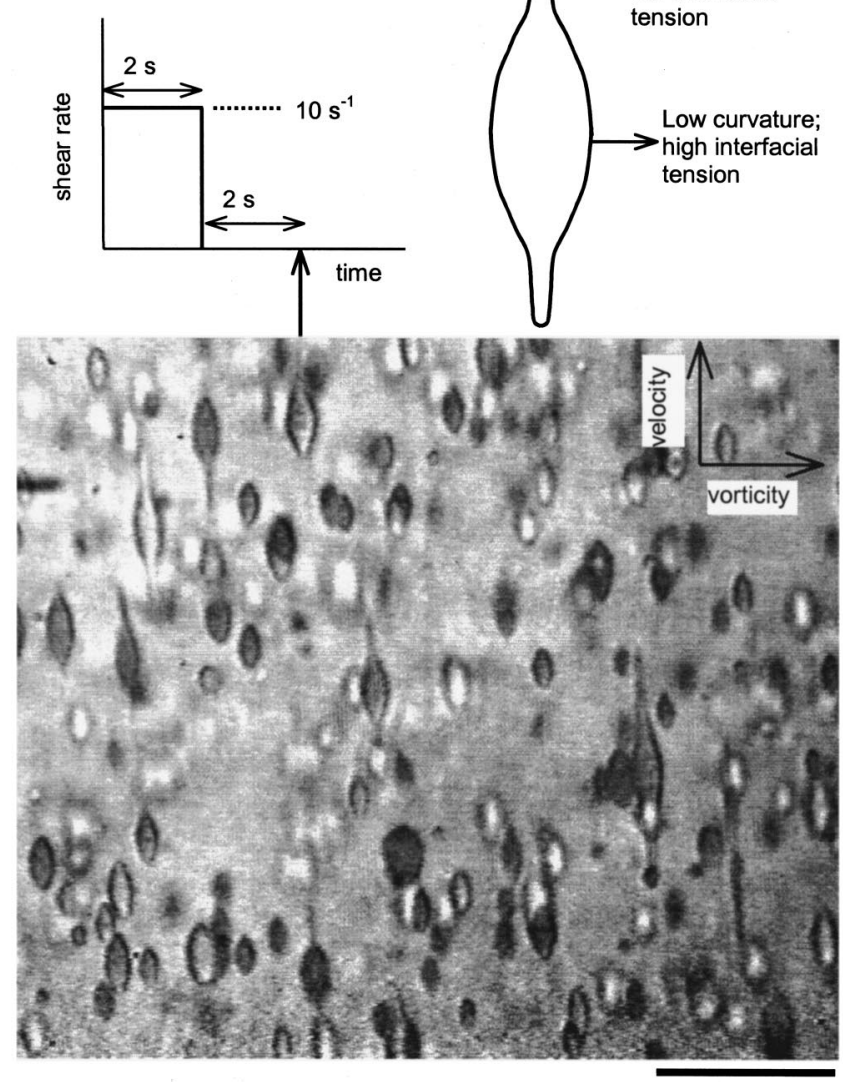

FIG. 5. Shapes of drops of the $2 \%$ compatibilized blend during relaxation following brief shearing at high shear rate. The thick line at the bottom right corner of the picture is $40 \mu \mathrm{m}$ in length.

the drop surfaces and lends support to the idea that Marangoni stresses act on droplet surfaces when the droplets are deformed by shear. Further support is obtained by the observation that droplets of the $10 \%$ compatibilized blend were found to deform very little under the same shear history indicating that the Marangoni stresses were sufficiently strong to prevent deformation altogether.

Qualitatively, the effects of Marangoni stresses on $\mathrm{Ca}_{\mathrm{cr}}$ are strongly analogous to those of an increased drop viscosity [Li and Pozrikidis (1997)]. Using this analogy, the magnitude of the Marangoni stress in the system may be estimated as follows: for the $2 \%$ compatibilized drops, $\mathrm{Ca}_{\mathrm{ss}} \approx 1$ (see Fig. 4). Past experimental work shows that the same $\mathrm{Ca}_{\text {cr }}$ would be obtained for an uncompatibilized drop if the viscosity ratio were either 0.025 or 2.5 [Grace (1982); Rallison (1984)]. The former value may be ignored as being physically unrealistic since an apparent increase in drop viscosity is expected in the present situation. Thus it may be concluded that the $2 \%$ compatibilized system behaves as though its viscosity ratio were 2.5 . The actual viscosity ratio is 1.06 , thus, the magnitude of the Marangoni stress is $\dot{\gamma} \eta(2.5-1.06) \approx 1.4 \dot{\gamma} \eta$. While exact numerical significance should not be assigned to this estimate, it is clear that the Marangoni stress is not a minor correction in the blends studied here; it is comparable to the "sensible" interfacial stress expected for drops with uniform interfacial tension. 


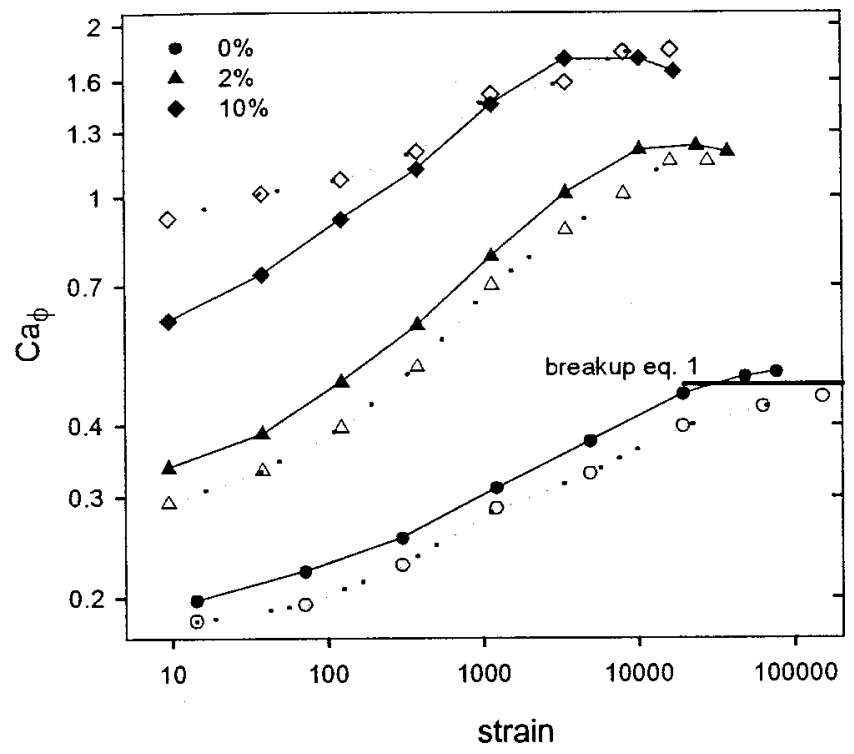

FIG. 6. Comparison of the evolution of capillary number $\mathrm{Ca}_{\phi}$ during coalescence for blends with $5 \%$ (open symbols, dotted lines) and 10\% (filled symbols, solid lines) by weight of dispersed phase. The legend shows the amount of compatibilizer as a weight fraction of the dispersed phase.

A final comment on the rate of coalescence is in order. Figure 3 shows that the time required to reach the steady shear capillary number decreases strongly with addition of compatibilizer. Optical microscopy has shown that the droplet size reached after preshear is greatly reduced upon addition of compatibilizer. Thus, the Smoluchowski frequency of binary collisions increases strongly due to the large increase in number density of droplets, as per Eq. (2). This is one possible cause for the faster approach to steady state, although other explanations may be possible. Regardless of the reasons, the increased rate of coalescence of compatibilized blends in Fig. 3 is not in doubt, which raises the following issue.

Steady shear is considered to be a dynamic equilibrium between the breakup and coalescence of droplets. If the coalescence is slow, the equilibrium is far towards the breakup side and all droplets are expected to be at or below the critical drop size. With an increase in the coalescence rate, the mean droplet size is expected to increase as the equilibrium shifts towards larger droplets. Thus, it is possible that the high steady shear capillary number is attributable not to a higher $\mathrm{Ca}_{\mathrm{cr}}$, but to a higher rate of coalescence, shifting the dynamic equilibrium between coalescence and breakup towards larger capillary numbers.

This alternative interpretation of the results may be tested by conducting experiments on blends with a lower volume fraction of the dispersed phase. This may be expected to reduce the droplet collision frequency significantly, and hence, reduce the coalescence rate. If the high steady shear capillary numbers for compatibilized blends were caused only by a higher coalescence rate, lowering the coalescence rate may be expected to decrease the steady shear capillary number as well. Accordingly, some coalescence experiments were performed on blends with $5 \%$ by weight of dispersed phase with $0 \%, 2 \%$, and 10\% compatibilizer (as a weight fraction of the dispersed phase as before). Figure 6 compares the evolution of $\mathrm{Ca}_{\phi}$ during coalescence for samples with $5 \%$ and $10 \%$ of dispersed phase. $\mathrm{Ca}_{\phi}$ rather than $\mathrm{Ca}$ is chosen for this comparison because the weak 
shoulder in $G^{\prime}$ for the $5 \%$ dispersed phase blends leads to large errors when fitting the Palierne model with a fixed $\phi$. It is seen that the steady state values of $\mathrm{Ca}_{\phi, s s}$ are almost equal for samples with $5 \%$ or $10 \%$ dispersed phase indicating that coalescence has negligible effect on the steady shear capillary number. This strongly supports our assumption that the steady shear capillary numbers correspond to the breakup capillary numbers $\mathrm{Ca}_{\mathrm{cr}}$.

\section{SUMMARY AND CONCLUSION}

This paper reports shear-induced coalescence experiments on immiscible polymeric blends with a droplet-matrix morphology. Dynamic mechanical measurements allowed direct determination of the average capillary numbers of the droplets. This corresponds to scaling the size of the compatibilized droplets to account for their lower interfacial tension. Addition of a surface-active compatibilizer increased the steady shear capillary number of droplets to values considerably greater than the $\mathrm{Ca}_{\mathrm{cr}}$ for breakup of uncompatibilized droplets. This suggests that the hydrodynamic stress required to break compatibilized droplets is considerably higher than that expected on the basis of their interfacial tension. It may therefore be concluded that the dynamics of the compatibilized blends studied in this paper cannot be explained merely in terms of their lower interfacial tension. Previous simulations [Stone and Leal (1990); Li and Pozrikidis (1997)] suggest that flow-induced gradients in the concentration of the compatibilizer on the droplet surface are responsible for an increase in $\mathrm{Ca}_{\mathrm{cr}}$ with compatibilizer concentration. These concentration gradients are believed to cause substantial gradients in interfacial tension (Marangoni stresses) along the interface that resist droplet deformation and breakup. Indeed, optical microscopy confirmed that compatibilized droplets deformed far less in shear flow than uncompatibilized ones. Microscopy experiments also provided direct evidence of gradients in interfacial tension along the surface of compatibilized droplets.

\section{ACKNOWLEDGMENTS}

P. V. P. is indebted to the FWO-Vlaanderen for a postdoctoral grant. S. V. thanks the Research Council at the K. U. Leuven for a postdoctoral fellowship. Financial support from the Research Council (GOA 98/06), K. U. Leuven is gratefully acknowledged.

\section{References}

Chesters, A. K., "The modeling of coalescence processes in fluid-fluid operations: A review of current understanding," Trans. Inst. Chem. Eng., Part A 69, 259-270 (1991).

Dalvi, M. C., C. E. Eastman, and T. P. Lodge, "Diffusion in microstructured block copolymers: Chain localizations and entanglements," Phys. Rev. Lett. 71, 2591-2594 (1993).

di Lorenzio, M. L. and M. Frigione, "Compatibilization criteria and procedures for binary blends: A review," J. Polym. Eng. 17, 429-459 (1997).

Dickie, R. A., "Heterogeneous polymer-polymer composites. I. Theory of viscoelastic properties and equivalent mechanical models," J. Appl. Polym. Sci. 17, 45-63 (1973).

Ferry, J. D., Viscoelastic Properties of Polymers (Wiley, New York, 1980).

Grace, H. P., "Dispersion phenomena in high viscosity immiscible fluid systems and applications of static mixers as dispersion devices," Chem. Commun. (Cambridge) 14, 225-227 (1982)..

Graebling, D., A. Benkira, Y. Gallot, and R. Muller, "Dynamic viscoelastic behavior of polymer blends in the melt-Experimental results for PDMS/POE-DO, PS/PMMA and POS/PEMA blends," Eur. Polym. J. 30, 301-308 (1994).

Graebling, D., R. Muller, and J. F. Palierne, "Linear viscoelastic behavior of some incompatible polymer blends in the melt. Interpretation of data with a model of emulsion of viscoelastic liquids," Macromolecules 26, 320-329 (1993). 
Gramespacher, H. and J. Meissner, "Interfacial-tension between polymer melts measured by shear oscillations of their blends," J. Rheol. 36, 1127-1141 (1992).

Guido, S., M. Simeone, and M. Villone, "Diffusion effects on the interfacial tension of immiscible polymer blends,"' Rheol. Acta 38, 287-296 (1999).

Hu, Y. T., D. J. Pine, and L. G. Leal, “Drop deformation, breakup, and coalescence with compatibilizer,” Phys. Fluids 12, 484-489 (2000).

Janssen, J. J. M., A. Boon, and W. G. M. Agterof, "Influence of dynamic interfacial properties on droplet breakup in simple-shear flow," AIChE J. 40, 1929-1939 (1994)..

Lacroix, C., M. Aressy, and P. J. Carreau, "Linear viscoelastic behavior of molten polymer blends: A comparative study of the Palierne and Lee and Park models," Rheol. Acta 36, 416-428 (1997).

Li, X. and C. Pozrikidis, "The effect of surfactants on drop deformation and on the rheology of dilute emulsions in stokes flow," J. Fluid Mech. 341, 165-194 (1997).

Lyu, S. P., F. S. Bates, and C. W. Macosko, "Coalescence in polymer blends during shearing experiments," AIChE J. 46, 229-238 (2000).

Macosko, C. W., P. Guegan, A. K. Khandpur, A. Nakayama, P. Marechal, and T. Inoue, "Compatibilizers for melt blending: Premade block copolymers," Macromolecules 29, 5590-5598 (1996).

Mewis, J., H. Yang, P. Van Puyvelde, P. Moldenaers, and L. M. Walker, "Small-angle light scattering study of droplet break-up in emulsions and polymer blends," Chem. Eng. Sci. 53, 2231-2239 (1998).

Milliken, W. J. and L. G. Leal, "The influence of surfactant on the deformation and breakup of a viscous drop: The effect of surfactant solubility," J. Colloid Interface Sci. 166, 275-285 (1994).

Milner, S. T. and H. Xi, "How copolymers promote mixing of immiscible homopolymers," J. Rheol. 40, 663-687 (1996).

Palierne, J. F., "Linear rheology of viscoelastic emulsions with interfacial tension," Rheol. Acta 29, 204-214 (1990).

Rallison, J. M., “The deformation of small viscous drops and bubbles in shear flows,” Annu. Rev. Fluid Mech. 16, 45-66 (1984).

Riemann, R. E., H. J. Cantow, and C. Friedrich, "Interpretation of a new interface-governed relaxation process in compatibilized polymer blends," Macromolecules 30, 5476-5484 (1997).

Stone, H. A. and L. G. Leal, “The effects of surfactants on drop deformation and breakup,” J. Fluid Mech. 220, 161-186 (1990).

Sundararaj, U. and C. W. Macosko, "Drop breakup and coalescence in polymer blends: The effects of concentration and compatibilization," Macromolecules 28, 2647-2657 (1995).

Vinckier, I., J. Mewis, and P. Moldenaers, "Relationship between rheology and morphology of model blends in steady shear flow," J. Rheol. 40, 613-632 (1996).

Vinckier, I., P. Moldenaers, A. M. Terracciano, and N. Grizzuti, "Droplet size evolution during coalescence in semiconcentrated model blends," AIChE J. 44, 951-958 (1998). 